\title{
DECIDABLE SEMIGROUPS
}

\author{
BY R. C. BUCK ${ }^{1}$
}

Communicated by Charles W. Curtis, March 21, 1968

An extension of the Burnside problem is to determine those presentations of semigroups which, by virtue of the nature of their defining relations, permit a decision algorithm and, in particular, are finite. One such result is that of Green and Rees [2]. The present note deals with another special class of semigroups.

Let $S_{n, r}$ be the semigroup generated by two elements $a$ and $b$, subject only to the relations:

$$
\begin{aligned}
& a b a=b^{n}, \\
& b a b=a^{r} .
\end{aligned}
$$

By symmetry, we may assume $n \leqq r$.

In [1], it was shown that the semigroups $S_{1, r}$ are each finite, with $\operatorname{ord}\left(S_{1, r}\right)=5 r+3$ for $r=1,2,3, \ldots$ In partial completion of this, we now have the following:

THEOREM. The semigroup $S_{2, r}$ is finite for $r=2,3,4$ with

$$
\operatorname{ord}\left(S_{2,2}\right)=31, \operatorname{ord}\left(S_{2,3}\right)=74, \text { and } \operatorname{ord}\left(S_{2,4}\right) \leqq 1390 \text {. }
$$

The semigroup $S_{2,5}$ is infinite, as are all the semigroups $S_{n, r}$ with $n \geqq 3$.

This result leaves undetermined the exact order of $S_{2,4}$, the nature of $S_{2, r}$ for $r \geqq 6$, and the question of whether a decision algorithm exists for the infinite semigroups; the case $n=2, r=5$ is somewhat special, and it may be possible that there are some that are finite with $r \geqq 6$.

We also note that the results in this theorem form an excellent test problem for persons interested in theorem proving by computer.

We outline only the proof of finiteness for the case $n=2, r=3$. The proof proceeds by deriving a sequence of further relations from (1) and (2), which suffice to reduce any word in $a$ and $b$ to one of the words in the following list:

1 Partially supported by the National Science Foundation. 
(3)

$$
\begin{aligned}
& a, a^{2}, a^{3}, \cdots, a^{16} \\
& b, b a, b a^{2}, b a^{3} \\
& b a^{2} b \\
& a b, a^{2} b, a^{8} b, \cdots, a^{13} b \\
& a b a, a^{2} b a, a^{3} b a, \cdots, a^{18} b a \\
& a b a^{2}, a^{2} b a^{2}, a^{3} b a^{2}, \cdots, a^{10} b a^{2} \\
& a b a^{3}, a^{2} b a^{8}, a^{3} b a^{3}, \cdots, a^{9} b a^{3} \\
& b a^{3} b, a b a^{3} b, a^{2} b a^{3} b, \cdots, a^{7} b a^{3} b
\end{aligned}
$$

Then, to prove that this list is irreducible (and the order of $S_{2,8}$ is therefore exactly 74), a representation of the semigroup is obtained as a collection of mappings on a finite set in which each word in (3) gives rise to a distinct mapping.

The following relations can be deduced from (1) and (2) when $n=2, r=3$ :

$$
\begin{aligned}
& a^{4}=b^{3} \\
& b a^{2} b a=a^{3} b \\
& a b a^{2} b=b a^{3} \\
& a^{k}\left(b a^{3} b\right) a^{k}=b a^{3} b \quad \text { for } k=1,2,3, \ldots \\
& a^{10} b a^{3}=a^{2} b a^{3} \\
& a^{14} b=a^{6} b \\
& a^{11} b a^{2}=a^{3} b a^{2} \\
& b a^{3} b a^{3} b=a^{10} b a^{2} \\
& b a^{3} b a^{3} b a^{3} b=a^{9} \\
& \left(a^{8} b\right)^{4}=a^{12} \\
& a^{17}=a^{9}
\end{aligned}
$$

An algorithm can then be described which uses these relations in a uniform way to reduce any word in $a$ and $b$ to one of the words listed in (3). To show that no word in this list can be transformed to any other word in the list by use of (1) and (2), a pair of mappings $f$ and $g$ is constructed which maps a set $X$ of 75 points into itself in such a way that $f \circ g \circ f=g \circ g$ and $g \circ f \circ g=f \circ f \circ f$. If $a$ is associated with $f$ and $b$ is associated with $g$, then each word $w$ in (3) corresponds to a mapping $\phi_{w}$.

The set $X$ is chosen as $\{0,1,2,3, \cdots, 74\}$. We introduce the special notation

$$
\left[c_{1}, c_{2}, \cdots, c_{k-1}, c_{k}^{*}, c_{k+1}, c_{k+2}, \cdots, c_{m}\right]
$$

to denote a mapping on $X$ such that 


$$
c_{1} \rightarrow c_{2} \rightarrow c_{3} \rightarrow \cdots \rightarrow c_{\uparrow} \rightarrow c_{k+1} \rightarrow \cdots \rightarrow c_{m}
$$

Using this, we can give succinct descriptions of the mappings $f$ and $g$ :

DeFinition of $f$.

$$
\begin{aligned}
& {\left[0,5,6,7, \cdots, 12,13^{*}, 14,15, \cdots, 19,20\right]} \\
& {\left[1,21,22,23,24,25,26^{*}, 27,28, \cdots, 32,33\right]} \\
& {\left[2,34,35, \cdots, 38,39^{*}, 40,41, \cdots, 45,46\right]} \\
& {\left[3,47,48,49^{*}, 50,51,52,53,54,55,56\right]} \\
& {\left[74,4,57,58^{*}, 59,60,61,62,63,64,65\right]} \\
& {\left[66^{*}, 67,68,69,70,71,72,73\right]} \\
& \text { Definition of } g .
\end{aligned}
$$

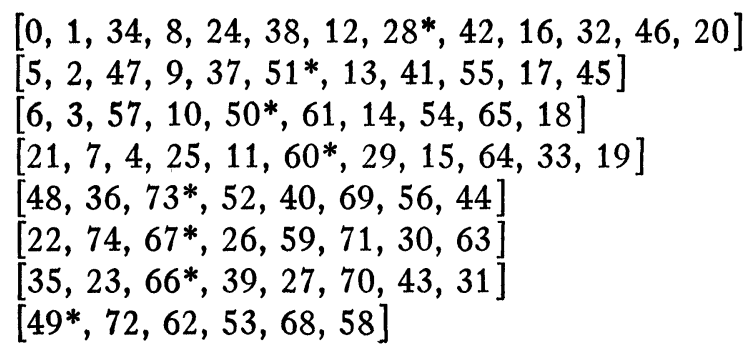

The mappings $\phi_{w}$ are shown to be all distinct simply by noting that each of the values $\phi_{w}(0)$ is different, and the case $n=2, r=3$ is completed.

The arguments for $n=2, r=2$ and $n=2, r=4$ are somewhat similar, although the derived identities are entirely different. In the case $r=4$, the crucial identity is $a^{125}=a^{25}$, which proves that the semigroup is finite. To show that $S_{2,5}$ is infinite, we add the relation $a b=b a$, and show that the resulting commutative semigroup is infinite. This technique does not work on the remaining cases, for it is easily seen that if $S_{n, r}$ is made commutative, the resulting semigroup is finite for every choice of $n$ and $r$ except $n=2, r=5$ and $n=r=3$.

\section{REFERENCES}

1. R. C. Buck, On certain decidable semigroups, Amer. Math. Monthly (to appear).

2. J. A. Green and D. Rees, On semigroups in which $x^{r}=x$, Proc. Cambridge Philos. Soc. 48 (1952), 35-40.

UNIVERSITY OF Wisconsin, MADISON 